\title{
A Petri Net Approach for the Analysis of VHDL Descriptions ${ }^{\star}$
}

\author{
S. Olcoz and J.M. Colom \\ TGI S.A. \\ Plaza Marqués de Salamanca 3-4, 28006 Madrid, Spain \\ Phone: $+(34) 13964925$. Fax: $+(34) 13964841$
}

\begin{abstract}
In this paper we propose a methodology for the detection of some bad behaviours (e.g. deadlocks) in VHDL descriptions based on structural analysis techniques of Petri nets. The bad behaviours that we consider concern the execution control flow of a VHDL description. This methodology works in three steps. First, a formal description in Petri Net terms of the execution control flow of a VHDL description is realized. We present the basic rules to perform this translation. Second, a. method to detect deadlocks using structural analysis techniques of Petri Nets based on structural invariants is applied. Finally, the information of the structural invariants allowing to decide the existence of a deadlock is used to fix the problem in VHDL terms.
\end{abstract}

\section{Introduction}

The possibilities of VLSI technology grows constantly and designs to be implemented on integrated circuits chips become more and more complex. Since 1960, the number of gates on a chip has doubled approximately every two years. As a consequence getting systems on to the market is becoming design limited instead of technology limited. Because the larger complexities, the amount of errors and undiscovered design bugs increase rapidly. Undetected errors has large consequences on costs if such designs are transformed into products. To cope with the problem of growing complexity design methodologies are being improved.

Inside these methodologies, formal verification during the design process is now growing acceptance, and can be viewed according to various approaches [1]. A first approach consist on validating partial properties of the proposed circuit implementation (e.g. safety or liveness properties). These properties play the role of partial specification and can be expressed, for example, by means of temporal logic. Another approach to formal verification refers to the proof of a given relationship between the implementation and the expected behavior, usually the implementation implies the specification.

In order to formally specifying hardware, some authors define new languages or calculi to describe the semantics of digital circuits allowing a formal proof

\footnotetext{
* This work has been supported by FORMAT project (ESPRIT III project N.6128).
} 
approach. For example, LCF-LSM [2], $\mu$ FP [3], FUNDS [4], etc. Instead of creating a new specific language, another authors propose to take advantage of the features of an existing Hardware Description Language (HDL), a priori more familiar to the designer. In this last case, in order to perform the formal verification a previous (automatic) translation from the HDL description into a mathematical formalism is required. The obtained model is verified by means of the specific calculus associated to the formalism. Examples of this approach can be founded in [5], [6] and [7].

The aim of this work is to investigate how formal verification techniques from Petri net theory [10] can be used to validate partial properties of a proposed HDL description. We consider as HDL the Very High Speed Integrated Circuit (VHSIC) Hardware Description Language (VHDL) [8] because is an industrial standard, but the method can be applied to other kind of HDLs.

On the other hand, VHDL provides a wide range of abstraction levels from the architecture level down to the gate level. The language is hierarchical and mixed-level simulation is supported. The concepts embodied in the timing model for the language mirror real hardware. In addition, VHDL provides a mechanism for building generic models which can be back-annotated with timing for specific devices. Many other excellent languages cover subsets of the capabilities that exist within VHDL, but none are as comprehensive. They do not cover the wide range covered by VHDL. An important gauge of the success of a HDL is its effectiveness as a design tool. Of prime importance is the fact that the VHDL language definition is independent of the tools which support it. Virtually every other language available today is tied closely with the tool which supports it, and in fact in most cases the definitions for these languages are ambiguous without the tool being available for interpretation. VHDL supports a wide spectrum of tools from simulation to synthesis to layout. The language has a concise specification and its controlled by the IEEE.

In order to validate properties of the design a method for automatic translation from VHDL description into a formal model to be proved is given. The internal formal model is a Petri net representing an abstract view of the VHDL descrition that only retains the VHDL source execution control flow and the signal exchange among processes. Taking into account that VHDL is primarily a simulation language, the VHDL's semantics is based on that of an discrete event simulation. Therefore, this simulation semantics for VHDL is also explicitly represented into the Petri net through the so called kernel process. The use of Petri nets as formal internal model allow us to make use of a well founded and mature theory of analysis [10] to validate a great amount of properties. Since the Petri net represents an abstract view of the VHDL description the kind of properties to validate are for example, mutual exclusion or absence of deadlock. There exist several analysis techniques of Petri nets. We will focus on that referred as structural analysis based on invariants.

We will focus on the verification of absence of deadlocks in the proposed description. So, we present a method to verify the property in the Petri net, based on invariants and a method to fix in the VHDL description the source of 
the problem (allowing to the designer correct the problem). We emphasize the fact that in the proposed approach the designer may have no expertise on the internal formalism nor the mechanisms involved in the proof process.

The paper is organized as follows. Section 2 recalls some definitions and notation concerning Petri Nets. Section 3 briefly describes the translation process from VHDL descriptions into Petri nets. Section 4 describes the algorithm to detect deadlocks and it is applied to an example. Section 5 draws some conclusions and future developments.

\section{Basic Definitions and Notation}

This section recall basic Petri net terminology and notation. The reader is referred to [10] for a nice tutorial on Petri Nets.

A place/transition net $\mathcal{N}$ is defined as a 4-tuple $\mathcal{N}=\langle P, T, P r e, P o s t\rangle$, where $P$ is the set of places $(|P|=n), T$ is the set of transitions $(P \cap T=\emptyset, P \cup T \neq \emptyset)$ $(|T|=m)$, Pre (Post) is the pre- (post-) incidence function representing the input (output) arcs Pre $: P \times T \rightarrow \mathbb{N}$ (Post $: P \times T \rightarrow \mathbb{N}$ ). Places and transitions are graphically represented by circles and bars, respectively.

The pre- and post-incidence functions can be represented as $n \times m$ matrices Pre and Post with elements Pre $\left[p_{i}, t_{j}\right]$ and Post $\left[p_{i}, t_{j}\right]$, respectively. The incidence matrix $C$ of the net is defined by $C\left[p_{i}, t_{j}\right]=\operatorname{Post}\left[p_{i}, t_{j}\right]-\operatorname{Pre}\left[p_{i}, t_{j}\right]$. We denote by $C[p]$ the row vectors of matrix $C$ (respectively) corresponding to place p. $C[t]$ is the column vector of $C$ corresponding to transition $t$.

A function $M: P \rightarrow \mathbb{N}$ is called a marking. A marking $M$ can be represented by a vector indexed by $P$. The marking of a place $p, M[p]$, is graphically represented by a number of tokens inside the place equal to $M[p]$. A marked net $\left\langle\mathcal{N}, M_{0}\right\rangle$ is a net $\mathcal{N}$ with an initial marking $M_{0}$.

A transition $t \in T$ is enabled at marking $M$ iff $\forall p \in P, M(p) \geq \operatorname{Pre}(p, t)$. A transition $t$ enabled at $M$ can fire yielding a new marking $M^{\prime}$ defined by $M^{\prime}[p]=M[p]-\operatorname{Pre}[p, t]+\operatorname{Post}[p, t]=M[p]+C[p, t] . M[t\rangle M^{\prime}$ denote that transition $t$ is enabled at $M$ and that $M^{\prime}$ is reached from $M$ by the firing of $t$.

A sequence of transitions $\sigma=t_{1} t_{2} \ldots t_{n}$ is a firing sequence of $\left\langle\mathcal{N}, M_{0}\right\rangle$ iff there exists a sequence of markings such that $M_{0}\left[t_{1}\right\rangle M_{1}\left[t_{2}\right\rangle M_{2} \ldots\left[t_{n}\right\rangle M_{n}$. The marking $M_{n}$ is said to be reachable from $M_{0}$ by firing $\sigma: M_{0}[\sigma\rangle M_{n}$.

$M[\sigma\rangle$ denotes a firable sequence $\sigma$ from marking $M$. The function $\bar{\sigma}: T \rightarrow \mathbb{N}$ is the firing count vector of the firable sequence $\sigma$, i.e. $\bar{\sigma}[t]$ represents the number of occurrences of $t \in T$ in $\sigma$. If $M_{0}[\sigma\rangle M$, then we can write in vector form $M=$ $M_{0}+C \cdot \bar{\sigma}$, which is referred to as the linear state equation of the net. A marking $M^{\prime}$ is said to be potentially reachable iff $\exists \bar{\sigma} \geq 0$ such that $M^{\prime}=M_{0}+C \cdot \bar{\sigma}$.

The reachability set $R\left(\mathcal{N}, M_{0}\right)$ is the set of all markings reachable from the initial one. Denoting by $P R\left(\mathcal{N}, M_{0}\right)$ the set of all potentially reachable markings we have: $R\left(\mathcal{N}, M_{0}\right) \subseteq P R\left(\mathcal{N}, M_{0}\right) . L\left(\mathcal{N}, M_{0}\right)$ is the set of all firing sequences and their suffixes in $\left\langle\mathcal{N}, M_{0}\right\rangle: L\left(\mathcal{N}, M_{0}\right)=\left\{\sigma \mid M[\sigma\rangle\right.$ and $\left.M \in R\left(\mathcal{N}, M_{0}\right)\right\}$.

A place $p \in P$ is said to be bounded iff there exists $k \in \mathbb{N}$ such that $\forall M \in$ $R\left(\mathcal{N}, M_{0}\right) M[p] \leq k$. A marked net $\left\langle\mathcal{N}, M_{0}\right\rangle$ is said to be (marking) bounded 
iff each of its places is bounded. A net $\mathcal{N}$ is structurally bounded iff $\forall M_{0}$ the marked nets $\left\langle\mathcal{N}, M_{0}\right\rangle$ are bounded. A transition $t \in T$ is live in $\left\langle\mathcal{N}, M_{0}\right\rangle$ iff $\forall M \in R\left(\mathcal{N}, M_{0}\right), \exists M^{\prime} \in R(\mathcal{N}, M)$ such that $M^{\prime}$ enables $t$. The marked net $\left\langle\mathcal{N}, M_{0}\right\rangle$ is live iff all its transitions are live (i.e. liveness of the net guarantees the possibility of an infinite activity of all transitions). The marked net $\left\langle\mathcal{N}, M_{0}\right\rangle$ is deadlock-free iff $\forall M \in R\left(\mathcal{N}, M_{0}\right) \exists t \in T$ such that $M$ enables $t$.

The left and right integer no negative annullers (p-semiflow and t-semiflow, respectively) of the incidence matrix leads to two kinds of structural invariants,

i) $Y \geq 0, \quad Y^{T} \cdot C=0 \Rightarrow Y^{T} \cdot M=Y^{T} \cdot M_{0}$ (token conservation law).

ii) $M\left[\sigma>M^{\prime}=M\right.$ (cyclic behaviour) $\Rightarrow X=\bar{\sigma}$ satisfies $C \cdot X=0$.

Another kind of linear invariants are those obtained from the integer nonnegative solutions $(Y \geq 0)$ of the system $Y^{T} \cdot C \Varangle 0$. From these solutions we obtain marking relations given by inequalities such as: $Y^{T} \cdot M \leq Y^{T} \cdot M_{0}$. The subset of places (transitions) corresponding to nonzero entries of a vector, $Y: P \rightarrow \mathbb{N}$ $(X: T \rightarrow \mathbb{N})$, is called the support and is denoted by $\|Y\|(\|X\|)$.

\section{Translating VHDL Descriptions into Petri Nets}

In this section we describe the key elements to express formally in terms of Petri Nets the execution control flow of a VHDL description. The execution model of a VHDL description is stated in the Language Reference Manual of VHDL [8] where the reader is referred for a detailed explanation.

The translation process we present take into account that in order to execute a model, the design hierarchy defining the model must first elaborated. Initialization of elaborated nets in the model then occurs. Finally, simulation of the model proceeds. Simulation consists of the repetitive execution of the simulation cycle, during which processes are executed and nets updated. Therefore, the execution model of VHDL descriptions contains the following basic elements: (a) A collection of user-defined processes consisting on sequential statements obtained from the design hierarchy through the elaboration process. Synchronization/communication among processes is based on the sequential signal assignment and wait statements. (b) A special process named kernel process which controls the time advancement and manages the communication among the userdefined processes through signals.

With this execution model in mind we develop the translation of the execution control flow of a VHDL description into Petri nets according to the following steps: 1) Translation of the sequential statements of VHDL; 2) Translation of the sequential signal and wait statements; and 3) Generation of the net corresponding to the kernel process. The rest of the section develops these steps.

Translation of the User-Defined Processes A VHDL description is composed by a set of concurrent statements structured in a design hierarchy. One of the most important concurrent statements is the process statement. This is 
because all concurrent statements have an equivalent process statement. We call these processes user-defined processes.

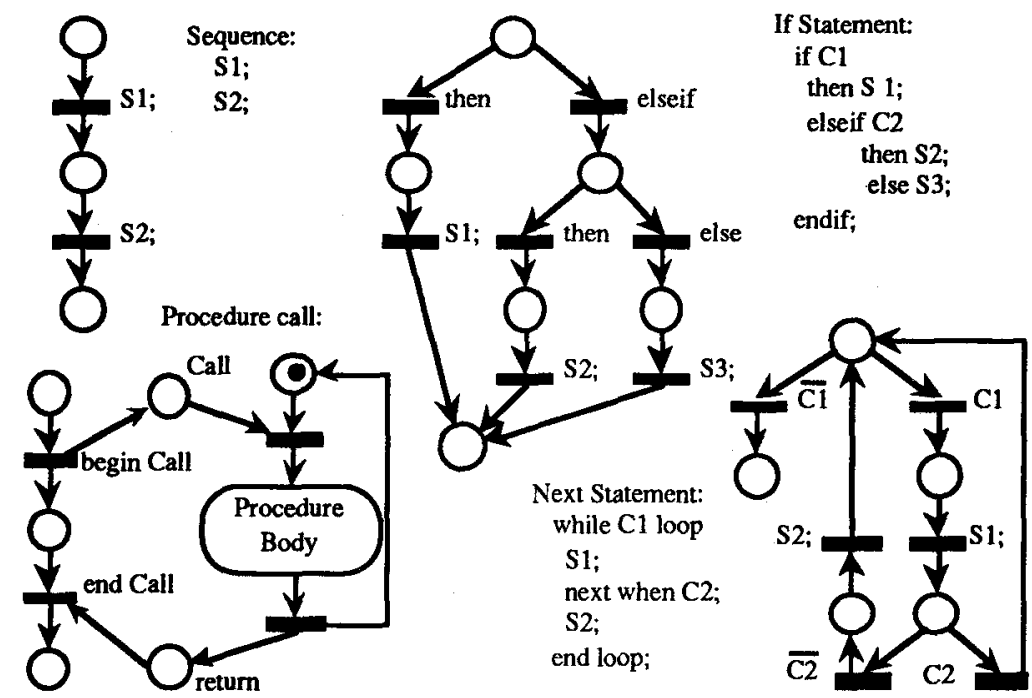

Fig. 1. Petri Net translation of the basics sequential control structures of VHDL.

The execution of a process statement consists of the repetitive execution of its sequence of statements. After the last statement in the sequence of statements of a process is executed, execution will inmediately continue with the first statement in the sequence. A proces can be in two different states: it can be active or suspended. A process communicate with other processes through signals. A process is suspended when it executes a wait statement, and is resumed when there is an event in the signals of the wait statement where it is suspended.

Therefore, it is obvious that we must focus on the description of the control flow corresponding to the different sequential statements. These descriptions give rise to subnets as those depicted in Fig. 1. All subnets have only one input place (representing the begin of the statement) and only one output place (representing the end of the statement). The different firing sequences moving a token from the input place to the output place, represent the different ways of executing a given sequential statement. The net corresponding to a process statement is built from the subnets corresponding to sequential statements by fussion of end places with begin places. The repetitive nature of a process statement is represented by means of the fussion of the end place of the last sequential statement with the begin place of the first sequential statement. A subnet corresponding to a process statement has as initial marking a token in the begin place of the first sequential statement. This initial marking represents the fact that in the initialization phase of the execution of a VHDL description, all processes are actives and execute 
statements until they reach a wait statement.

The translation rules for process statement can be directly applied to the translation of the execution control flow of subprograms. This is because, in essence, the procedure body is a piece of sequential code. Nevertheless, an additional aspect must be considered referred to the procedure call/return mechanisms. These mechanisms are expressed by means of two places (see in Fig. 1 the corresponding subnet). Place call represents the activation of the procedure while place return represents the end of the procedure execution (if a subprogram has different exit points each one must be connected to place return).

Translation of Sequential Signal Assignement and Wait Statements. A signal assignement statement modifies the projected output waveforms contained in the drivers of one signal. On the other hand, the wait statement causes the suspension of a process or a procedure. Through these two statements, a given process can communicate/synchronize with another one. In Fig. 2 we depict the subnet associated to two simple wait and signal assignment.

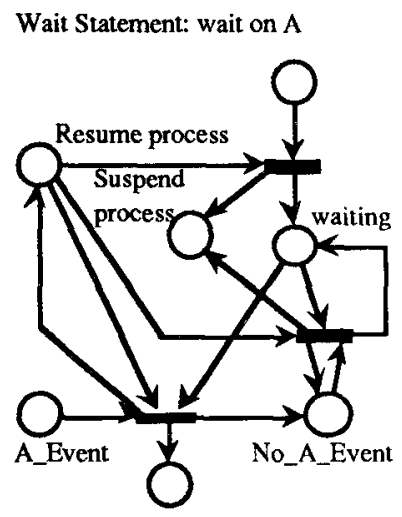

$$
\text { Signal Assignement Statement: } \mathrm{A}<=\mathrm{B} \text {; }
$$

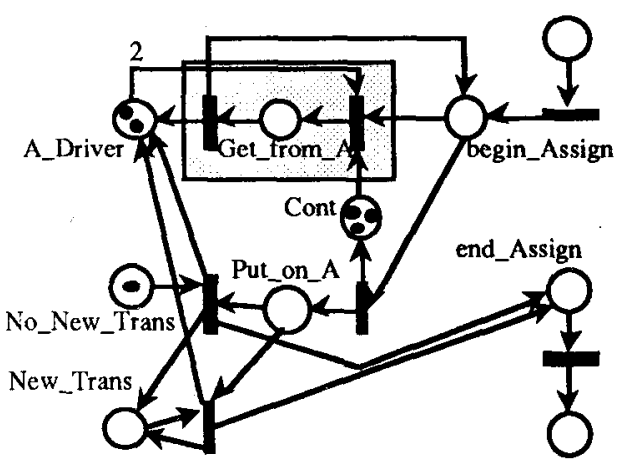

Fig. 2. Petri Net translation of simple wait and signal assignment statements.

In the subnet of the wait statement we can observe that the first operation is to change the process from the active state to the suspended one. Each time the kernel process actives the process containing this wait statement (a token in the place Resume_process) the subnet try to fire one of the output transitions of the waiting place. If there exists an event (a token in place A_Event) on A then the process is resumed, otherwise (a token in place No_A_Event) the process is again suspended.

In the subnet associated to the signal assignment we represent: (1) The generation of a new transaction that is added (place Put_on_A) to the corresponding driver; and (2) The removing (place Get_from_A) of some old transactions (from place A_Driver) according to the timing model (preemption of transactions). 
This last operation only represents a possibilistic model where we model the net balance of pending transactions. In the case in which there is no preemption of transactions in the driver, this part of the subnet can be removed.

Generation of the kernel process The kernel process is a conceptual representation of the agent that coordinates the activity of the user-defined processes during the execution. This agent causes the propagation of signal values of implicit signals to be updated. Furthermore, this process is responsible for detecting events that occur and for causing the appropiate processes to execute in response to those events.

Subnets of the user-defined processes communicate with the kernel process through three kind of places: Places representing drivers of signals, Places representing events on (implicit and explicit) signals, Control places to resume and suspend a process. All of these places are managed by the kernel process. This process carry out the simulation cycle that is executed repetitively until there were no pending events. It is composed by the following stages (see an example of the structure of this net in Fig. 5 corresponding to the example developed in section 4): (1) Initially, all resume places corresponding to all present processes are marked and then all of them can be executed concurrently in an asynchronous way; (2) The kernel process waits for a token in each suspend place of each user-defined process. Observe that the kernel process will not authorize a new simulation cycle until all the processes were suspended; (3) The kernel process updates the signals and schedules all events needed in the next simulation cycle in order to resume (if possible) all processes now suspended on wait statements. For each event it schedules a copy to each process that can consume it.

\section{An Example}

In this section we present a simple example to illustrate the translation of a VHDL description into a Petri Net, and the analysis of the net in order to detect possible anomalies in it. The proposed example contains two communicating processes. The first, called $C O M P$, compute the square of a natural number $(A)$ that it receives as input. The result $(P)$ is sent to the other process, called $I N P U T$, which obtains from the keyboard the integer $A$, waits for the result $(P)$ given by COMP and represents $P$ in the screen. The basic skeleton of the description to analyze (only the statements related with the execution control flow) is depicted in Fig. 3.

To translate the description into a Petri Net, we have divided the whole net into three subnets: the associated to the processes INPUT and COMP, (Fig. 4), and the associated to the KERNEL process (Fig. 5). In order to do cleaner the three nets we have replicated some places in the figures. They are distinguished because they have the same names. To compose the subnets we must merge the places with the same names.

To translate the process INPUT we must concatenate the subnets corresponding to a signal assignment statement and a wait statement as depicted in 


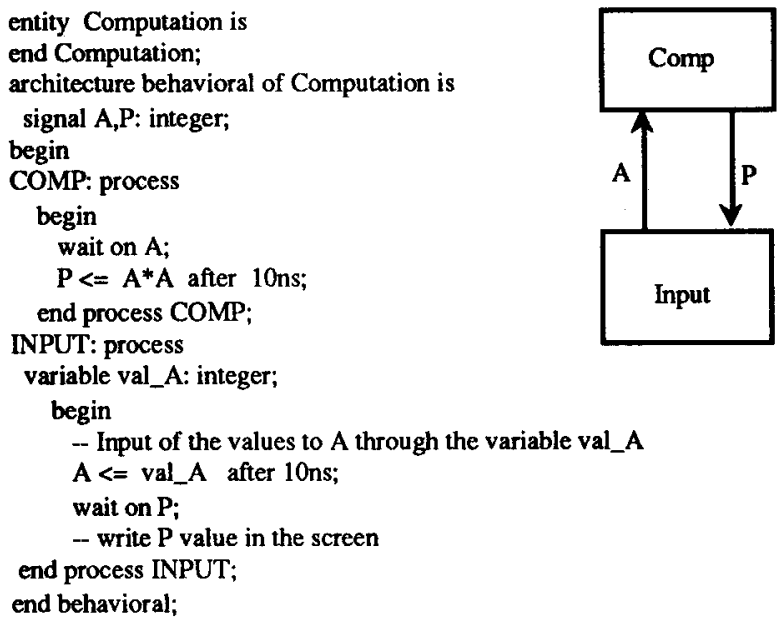

Fig. 3. A simple VHDL Program.

Fig. 4. Observe, that the assigment is simpler than presented in Fig. 2, this is because all transactions generated remain into the driver (place $A_{-}$Driver) until they are scheduled (there is no preemption). To exit from the wait statement an event on $P$ is needed, and this happens when place $P_{-} E v e n t$ receives a token (observe that places $P_{-}$Event and $N o_{-} P_{-}$Event are in mutual exclusion, i.e., they are not marked simultaneously). Places $N o_{-} N$ New_Trans and New_Trans are enclosed to retain if a new transaction has been generated in the actual iteration (observe that this places appear also in the COMP process). The translation of process $C O M P$ is very similar to the $I N P U T$ process, taking into account the events and drivers that are implied.

Both processes are repetitive, alternating between resume and suspend states. This is represented through places Resume_Input, Resume_Comp, Suspend_Input and Suspend_Com. These places are updated by the processes. When both processes are in the suspended state the kernel process can go (firing of transition $T K 2$ ) to update the drivers and to schedule all events on the implicit signals. This is represented by the two subnets between transitions $T K 6$ and $T K 19$. For example, in case of the driver of $P$, first we decide if a pending transaction in the $P \_$Driver place must be scheduled (transitions $T K 7$ and $T K 8$ ). If a transaction is scheduled then we must decide if an event is scheduled (transitions $T K 11$ and $T K 12$ ). If an event on $P$ is scheduled, then the place $P_{-}$Event is marked indicating that a new event can be consumed for the wait statement in process INPUT, otherwise place $N_{-} P_{-} E v e n t$ remains marked and then the process INPUT will go again to the suspended state (firing transition 15 ). Places New_Event and No_New_Event are used to indicate if at least (or not) a new event on the implicit or explicit signals must be processed. If this is the case then transition $T K 21$ will be fired and the kernel process will resume all suspended processes (firing $T K 1$ ), otherwise transition $T K 20$ fres and the kernel process try again 


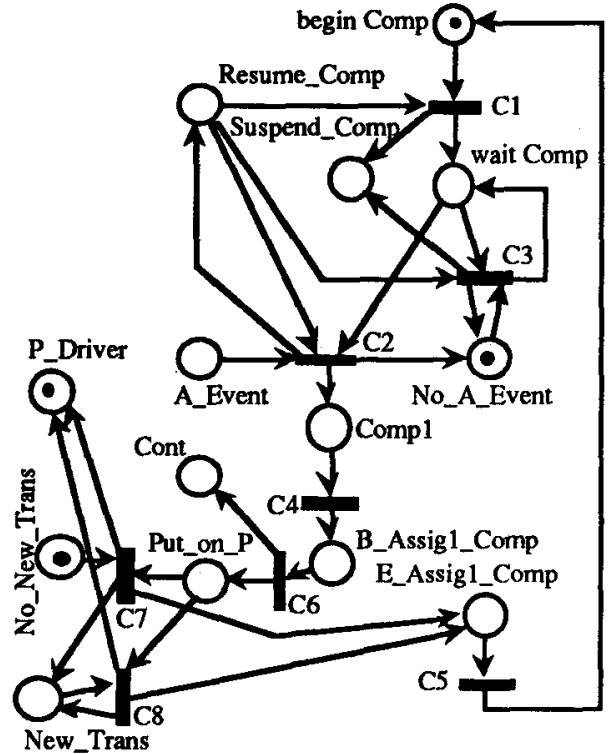

COMP Process

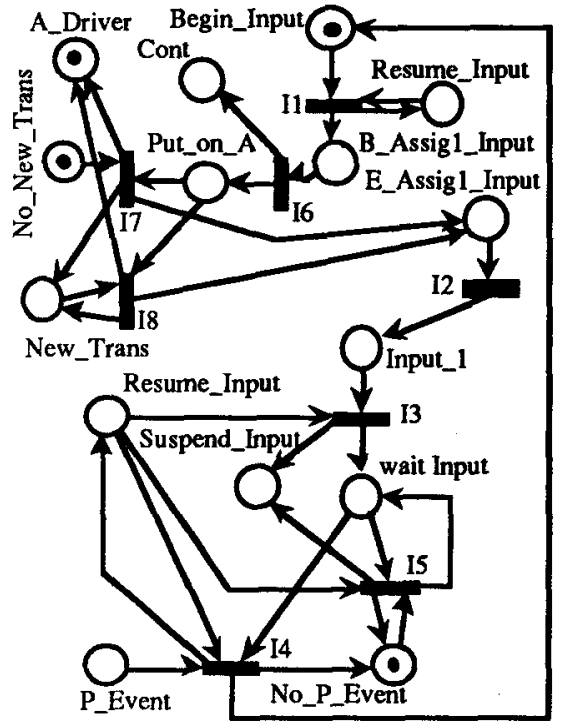

INPUT Process

Fig. 4. Petri Nets associated to COMP and INPUT Processes.

with the transactions contained into the drivers in order to schedule events. This process continues until there is no pending transactions (deadlock on transition $T K 5$, because place Cont that retains the global number of pending transactions contains zero tokens).

With the Petri Net obtained from the VHDL program now we illustrate the use of this model to analyze properties of the VHDL program. The property we analyze is the deadlock freeness. This is because according to the initial specification of the VHDL description, it must be execute forever.

The method we apply to detect and remove some potentially reachable deadlocks is only based on structural analysis techniques of Petri Nets. Obviously, with this method we can not detect all kinds of deadlocks (e.g. deadlocks depending on the signals value, because we do not represent the data in the Petri Net). Therefore, we only obtain a necessary condition on the infinite execution of the program. Nevertheless, the structural analysis techniques are very efficient from a computational point of view and they are specially indicated in previous phases of the design. If we want a necessary and sufficient condition on the proposed property we must apply techniques based on the reachability graph.

The method we propose is based on the analysis of marking invariants of the net. If we compute the p_invariants of the net corresponding to our program, we can observe that there are no pinvariants containing places which represent events or transactions. This means that the number of tokens inside these places does not accomplish any token conservation law. This can be a source of problems 


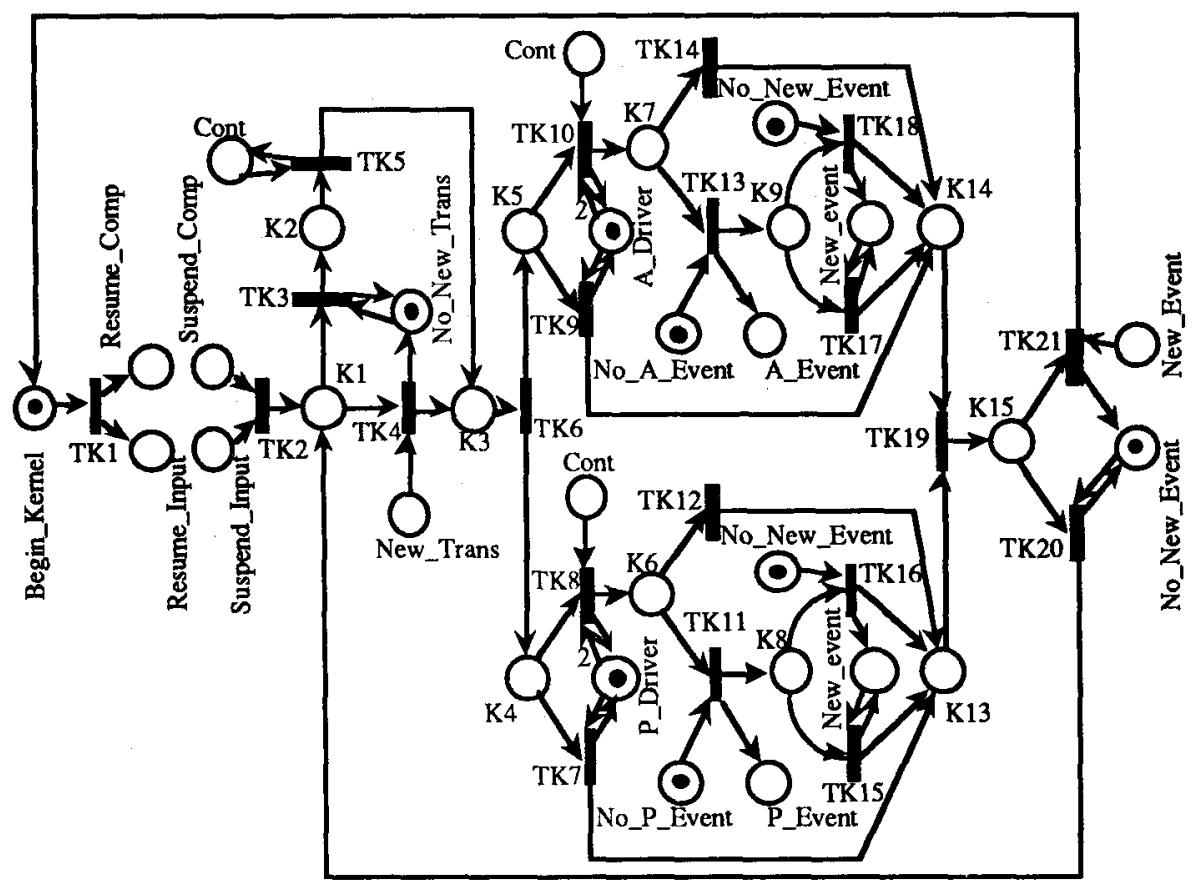

Fig. 5. Petri Net associated to KERNEL Process of the example program.

since if these places loose tokens in a continued way, then they can deadlock their output transitions.

To analyze this situation, from a structural perspective, we compute marking invariants such as $Y^{T} \cdot M \leq Y^{T} \cdot M_{0}$, where $Y$ is computed from the system of inequalities $Y^{T} \cdot C \lessgtr 0$. These invariants express the following fact: if we fire continously certain transitions, we decrease continously the total contents of tokens of places $\|Y\|$ and then some output transitions of these places will be deadlocked. In order to see this we premultiply the net state equation by the vector $Y: Y^{T} \cdot M=Y^{T} \cdot M_{0}+Y^{T} \cdot C \cdot \bar{\sigma}$. Since $Y^{T} \cdot C \lessgtr 0$, some entry of $Y^{T} \cdot C$ is negative and the firing of the corresponding transition always decrease the contents of places $\|Y\|$. These transitions will be called sink transitions. If we compute for our example these invariants we obtain two of them:

$\mathrm{M}\left(\mathrm{P} \_\right.$Driver $)+\mathrm{M}(\mathrm{K} 6)+\mathrm{M}\left(\mathrm{P} \_\right.$Event $)+\mathrm{M}$ (A_Driver $)+\mathrm{M}(\mathrm{K} 7)+\mathrm{M}$ (A_Event) $+\mathrm{M}$ (Begin_Input) $+\mathrm{M}$ (B_Assig1_Input $)+\mathrm{M}$ (Put_on__A $)+\mathrm{M}($ Comp1 $)+$ $\mathrm{M}($ B_Assig1_Comp $)+\mathrm{M}($ Put_on_P $) \leq 3$

$$
\begin{aligned}
& \mathrm{M} \text { (Cont) }+\mathrm{M}(\mathrm{K} 6)+\mathrm{M}(\mathrm{K} 7)+\mathrm{M}\left(\mathrm{P} \_ \text {Event }\right)+\mathrm{M} \text { (A_Event) }+\mathrm{M} \text { (Begin_Input) } \\
& +\mathrm{M} \text { (B_Assig1_nput) }+\mathrm{M}(\text { Comp1) }+\mathrm{M} \text { (B_Assig1_Comp }) \leq 1
\end{aligned}
$$

The sink transitions of these invariants are $T K 12$ and $T K 14$ (Fig. 5). It means if all transactions are consumed and no event is generated (i.e., firing 
$T K 12$ and $T K 14)$ then the net is deadlocked. In other words, there is a mistake in the original program in the wait statement of COMP process, because the process is suspended waiting for an event on $A$, but is possible we receive a duplicate of the $A$ value and then no event will be generated and therefore the two processes will be deadlocked in their respective wait statement.

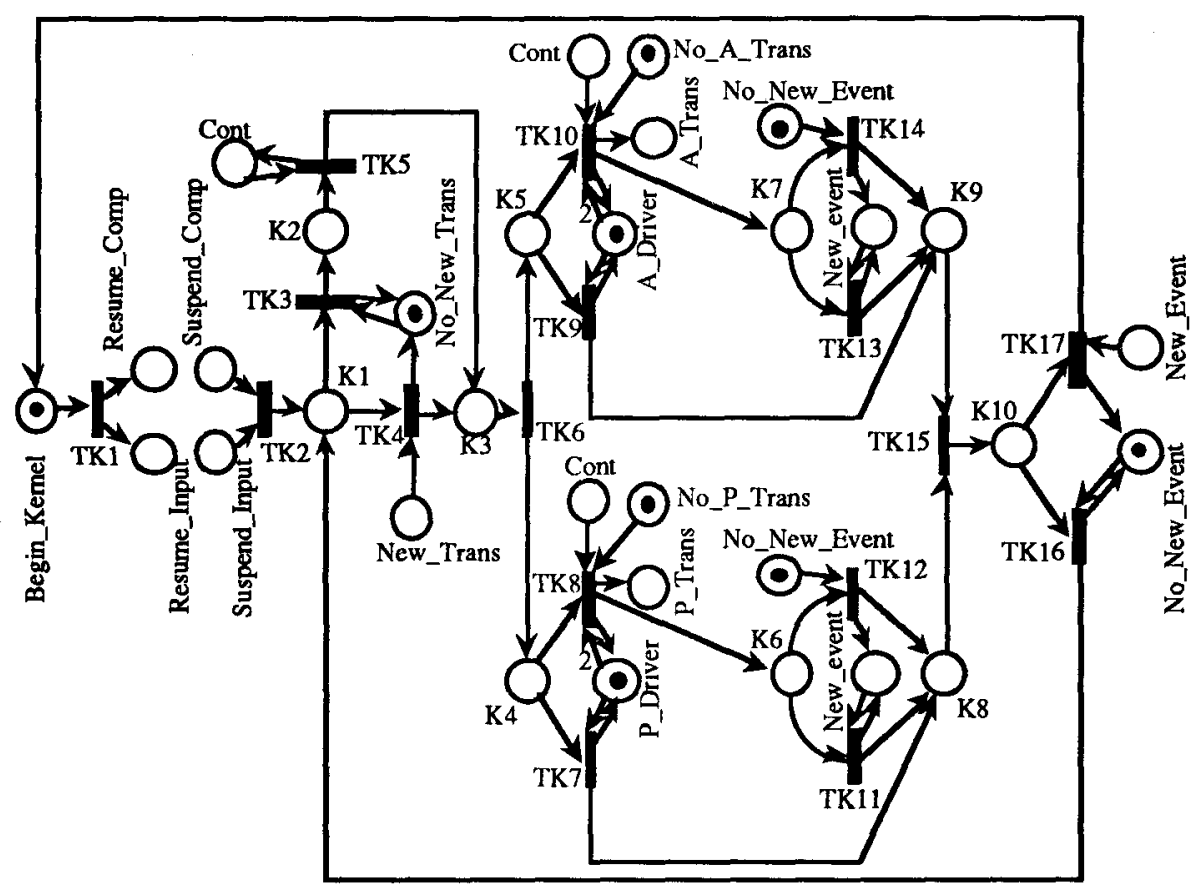

Fig. 6. Petri Net associated to KERNEL Process of the corrected example program.

In order to solve this problem we must change the statement of process COMP, "wait on A;", for the statement "wait on A'Transaction". The translation of the new description gives rise to the same nets of Fig. 4 (but changing the names of the places A_Event, No_A_Event, P_Event and No_P_Event by the names $A_{-}$Trans, No_A_Trans, P_Trans, No_P_Trans respectively). The net corresponding to the new kernel process is depicted in Fig. 6 (where we have assumed a new wait statement for process INPUT: "wait on P'Transaction;"). If we repeat the above analysis to this new net, we obtain that all places belong to at least one p_invariant. In order to verify exactly that the net is deadlock free then we can apply an analysis based on the reachability graph. And in this case the result is deadlock free. 


\section{Conclusions}

In this paper we have shown that Petri Nets are an useful formal model to analyze properties (of interest for the designer) of a VHDL description. The approach presented starts with a translation of the VHDL description into a Petri Net. In order to do this we have defined the subnets associated to each sequential statement that can appear inside a process statement (all the other concurrent statements are considered through its equivalent process). The obtained net only retains the aspects related with the execution control flow of the VHDL description. The generated net contains a process for each VHDL process plus a special process, called kernel process, through which we represent the simulation cycle of a VHDL description.

As the example of section 4 has shown, the obtained nets from a VHDL program have a great size. Therefore, in order to do viable the use of the proposed approach, the generation of the net must be integrated into a CAD framework. This can be easily done from the simple translation rules presented in the paper.

From the obtained net, several analysis techniques can be applied in order to verify many properties of interest. In the paper we have shown that the use of structural analysis techniques is very interesting because the computational efficiency of the analysis algorithms and because the great amount of information that can be extracted from the analysis of the results in order to correct possible errors in a description. So, in the example we have detected the presence of deadlocks in the net through a special kind of marking invariants. Moreover, interpreting these invariants we have found the source of the problem in the VHDL description.

\section{References}

1. P. Camurati, P. Prinetto: Formal verification of Hardware Correctness: Introduction and Survey of current Research. IEEE Computer, July 1988, pp 8-19.

2. M. Gordon: LCF-LSM. Technical Report N. 41, University of Cambridge (UK), 1984.

3. M. Sheeran: $\mu \mathrm{FP}$, a Language for VLSI design. Proc. ACM Symposium on LISP and Functional Programming, Austin, Texas (USA), 1984.

4. R. Boute: System Semantics: principles, applications and implementation. ACM Transaction on Programming Languages and Systems, Vol. 10, N. 1, January 1988.

5. A. Bartsch et al.: LOVERT - A logic verifier of Register-Transfer Descriptions. Proc. IFIP International Workshop on Applied Formal Methods for Correct VLSI Design, Houthalen (Belgium), November 13-16, 1989.

6. R. Piloty et al.: CONLAN Report, Springer Verlag, 1983.

7. J. Vantassel et al.: Toward a formal verification of VHDL specifications. Proc. IFIP W.G. 10.2 International Workshop on Applied Formal Methods for Cornect VLSI Design, Houthalen (Belgium), November 13-16, 1989.

8. IEEE Standard VHDL Language Reference Manual, IEEE, Inc. New York, NY, March, 1988.

9. T. Murata: Petri nets: properties, analysis, and applications. Proceedings of the IEEE, Vol. 77, No. 4, April 1989, pp 541-580. 